\title{
Secure e-commerce web development framework
}

\begin{abstract}
This study presents a framework for safety critical e-commerce application development based on an Extreme Programming methodology with inbuilt security across the development lifecycle to mitigate security lapses. This approach tightens security checks and balances at every stage of development process, minimizes any vulnerability that will manifest in production environment and avoids an unnecessary extension of the life cycle.
\end{abstract}

Keyword: Development framework; E-commerce; Extreme programming; Web engineering; Web security 